\title{
Prevenção universal e promoção de saúde em grupo de crianças a partir do Método Friends
}

\author{
Giulia Tatiana Tkaczyk Pavoski \\ Caroline Guisantes de Salvo Toni \\ Ana Priscila Batista \\ Cynthia Lais Ignachewski \\ Universidade Estadual do Centro-Oeste, Paraná, Brasil
}

\begin{abstract}
Resumo
Os transtornos de ansiedade e depressão têm aumentado de forma considerável na população, sendo que seus sintomas tendem a manifestar-se já na infância. Intervenções para esses transtornos podem ser realizadas através do desenvolvimento de habilidades socioemocionais e de resiliência. O Método Friends é um programa baseado em evidências que visa desenvolver essas habilidades. Nesse contexto, esta pesquisa consistiu em uma avaliação da eficácia do Método Friends em um grupo de crianças. A amostra consistiu de 10 crianças, de ambos os sexos, com idades entre 6 e 7 anos. Estas participaram do Método em uma Clínica-Escola de uma cidade do Interior do Paraná. Foi utilizado como instrumento de avalição o CBCL, aplicado em três momentos: pré-teste, pós-teste e follow up. Foram verificadas diferenças estatisticamente significativas nos três momentos em escalas relacionadas a competências sociais, escala de internalização e de externalização, ansiedade, depressão, retraimento e comportamento agressivo. De forma geral observou-se a eficácia do Método.

Palavras-chave: Iinfância; Ansiedade; Depressão; Resiliência; Psicologia preventiva.
\end{abstract}

\section{Universal prevention and promotion of health in a children's group from the Friends Method}

\begin{abstract}
Anxiety and depression disorders have been considerably increasing in the population and their symptoms tend to show up since childhood. Interventions programs for these disorders can be done through development of sociomeocional and resilience skills. Friends Method is an evidence-based program that aims to develop these skills. Thus, this study aimed to assess the Friends Method effectiveness in children groups. The sample consisted of 10 children, both gender, aged between 6 and 7 years old. The study took place in a University Clinic school in Parana, Brazil. CBCL was used as instrument, had been applied in three moments: prettest, posttest and follow up. It was found significantly statistic differences among the three phases of data collection according to scales related to social, Internalizing Problems and Externalizing Problems scales, anxious and depressed, withdrawn and depressed, agressive behavior. The effectiveness of the Friends Method was observed.
\end{abstract}

Keywords: Childhood; Anxiety; Depression; Resilience; Preventive psychology.

\section{Universal prevención y promoción de la salud en un grupo de los ninos de lo Método Friends}

\section{Resumen}

Los índices de ansiedad y depresión tiene aumentado de forma alarmante en la populación, y sus síntomas tiendem a manifestarse ya en la infancia. Intervenciones sobre eses transtornos puedem ser realizados a través del desarrollo de las habilidades socioemocionales y de la resiliencia. El Método Friends es uno programa basado en evidencias que visan desarrollar esas habilidades. Nesse contexto, esta investigación consistiu en una avaliación de la eficacia del Método Friends en uno grupo de niños. Los participantes fueron 10 niños, de ambos los sexos, con idades entre 6 y 7 años. Estos participaron del Método en una Clínica Escuela de una ciudad del interior del Paraná. Fue utilizado como instrumentos (G1) de la avaliación el CBCL, aplicado en tres momentos: pretest, postest y follow up. Fueran verificadas diferiencias estaticamente significativas en los tres momentos en escalas relacionadas a las competencias sociales, escalas de internalização e externalização, ansiedad, depresión, retraimiento y comportamiento agresivo. De forma general observouse la eficacia del Método.

Palabras clave: Infancia; Ansiedad; Depresion; Resiliencia; Psicología preventiva. 


\section{Introdução}

Relatórios mundiais da Organização Mundial de Saúde (WHO 2001, 2004, 2012) apontam que a incidência de psicopatologias como os transtornos de ansiedade e os transtornos depressivos tem aumentado de forma alarmante nos últimos anos. Além disso, esses relatórios alertam que eles representarem alto custo social e econômico, estando entre as doenças mais incapacitantes do mundo. Estudos como de Stallard, Skryabina et al., (2014), Kessler et al., (2007), Iizuka e Barrett (2011), apontam que os primeiros sinais de transtornos mentais aparecem antes dos 14 anos, sendo que os sintomas de rastreio da ansiedade e depressão podem ser observados já na infância. Nesse contexto, intervenções que previnam tais doenças mentais tornam-se relevantes. O Método FRIENDS é um programa estruturado que tem demonstrado capacidade de prevenção de ansiedade e depressão na infância, além do desenvolvimento de resiliência em seus participantes (Iizuka \& Barrett, 2011), sendo utilizado em vários países (Iizuka \& Barrett, 2011; Figueredo, no prelo, Kösters et al., 2012). No Brasil, ainda não existem pesquisas avaliando sua eficácia. Diante disso, o objetivo deste estudo foi a avaliação da eficácia do Método FRIENDS, em um grupo de crianças com idades entre de 6 e 7 anos de uma cidade do interior do Paraná. Melnik (2011) afirma que o conceito da eficácia busca descrever a capacidade de uma intervenção produzir os resultados esperados para um determinado grupo de participantes. Já a efetividade descreve a capacidade de generalização da intervenção, ou seja, o quanto essa é capaz de produzir os mesmos bons resultados em locais e grupos diferentes (Melnik, 2011).

Intervenções eficazes de prevenção a transtornos de ansiedade e depressão na infância são relevantes, visto os grandes prejuízos pessoais e sociais que estes transtornos acarretam (Papalia \& Feldman, 2013; Vianna, Campos, \& Fernandez, 2009, Bahls, 2002). $\mathrm{O}$ transtorno depressivo pode ser caracterizado por humor triste e vazio. Esta mudança no funcionamento do sujeito pode ser acompanhada por alterações cognitivas e somáticas (APA, 2014). O transtorno depressivo na infância caracteriza-se por sintomas que incluem incapacidade de se divertir ou se concentrar, fadiga, atividade extrema ou apatia, queixas somáticas, irritabilidade, ansiedade, choro frequente, alterações de sono, alteração de peso, movimentos repetitivos, tristeza, sentimentos de inutilidade, sensação prolongada de falta de amigos, o distanciamento social, a baixa autoestima, e/ ou pensamentos frequentes sobre morte ou suicídio (Papalia \& Feldman, 2013; Bahls, 2002; APA, 2014).
Tem-se observado que a incidência dessa patologia tem aumentado na infância (WHO, 2011, 2012, 2014). Porém, os estudos sobre prevalência da depressão infantil ainda são escassos no Brasil e provavelmente os índices são subestimados (Fraga, 2015), sendo que o único estudo brasileiro (Bahls, 2002) aponta uma prevalência de transtorno depressivo maior de 0,4 a $3,0 \%$ em crianças e de $3,3 \%$ a $12,4 \%$ em adolescentes.

Os transtornos de ansiedade são também frequentes em crianças e adolescentes. A ansiedade pode ser definida como uma resposta buscando a autoproteção com foco no medo e em uma preocupação excessiva e intensa da criança; ainda se apresenta como uma angústia de algo que está para acontecer, ou que ela acha que vai acontecer (Stallard, 2010). Segundo Vianna, Campos e Fernandez (2009), os transtornos de ansiedade podem acarretar consequências de médio a longo prazo para a vida do sujeito como, por exemplo, prejuízos escolares, sociais, abuso de medicamentos e drogas e até, posteriormente, transtornos depressivos. Os autores apontam que o dado mais recente sobre a incidência dos transtornos de ansiedade no Brasil é de que $3,4 \%$ das crianças e $5,04 \%$ dos adolescentes apresentam algum transtorno de ansiedade.

Tendo isso em vista, é importante ressaltar a necessidade de mais intervenções e práticas envolvendo prevenção e promoção de saúde focadas na infância, visto que são observados os sintomas de rastreio de diversas psicopatologias no início dessa fase (Iizuka \& Barrett, 2011). Segundo Lohr, Pereira, Andrade e Kirchner (2007), a prevenção tem como objetivo a minimização dos fatores de risco para uma doença buscando a construção de intervenções antes do seu surgimento. Segundo essas autoras, o campo da prevenção tem crescido na ciência psicológica, tanto por seus resultados positivos a médio e longo prazo, quanto pelos custos baixos para a sua aplicação.

As ações preventivas, como afirmam Abreu, Barletta e Murta (2015), têm como principal objetivo o aumento de fatores protetivos e a diminuição dos riscos do surgimento de alguns problemas. Quando se trata de prevenção, pode-se dividi-la e classifica-la em três níveis: prevenção primária, prevenção secundária e prevenção terciária. Segundo Lohr, Melo, Salvo e Silvares (2013), a prevenção primária se caracteriza pela promoção de saúde para evitar que alguma doença venha a surgir; já a prevenção secundária diz respeito ao diagnóstico de uma doença e seu tratamento precocemente para que diminua seus efeitos ou até mesmo a elimine; por fim, a prevenção terciária se caracteriza pela reabilitação quando a doença já está instalada. 
As práticas focadas em prevenção primária têm ganho especial atenção no âmbito da saúde mental. Segundo Abreu et al. (2015), a prevenção primária pode ser subdividida em prevenção universal (intervenções para toda a população); prevenção seletiva (intervenções para pessoas que estão expostas a fatores de riscos para sua saúde); e prevenção indicada (intervenções para pessoas que apresentam sintomas precoces de um determinado transtorno, mas que não possuem um diagnóstico sobre ele). As práticas de prevenção universal têm sido amplamente defendidas, em especial no âmbito da Saúde Mental, visto a interrelação entre a prevenção e a promoção de saúde: são necessárias intervenções para que se possam prevenir futuros transtornos, reduzindo fatores de risco, mas que também promovam habilidades, aumentem os fatores de proteção e construam recursos no repertório do sujeito. "A redução em fatores de risco só é possível se os fatores protetivos foram maximizados" (Abreu et al., 2015, p. 67). Ou seja, a partir de práticas de promoção de saúde a Psicologia pode auxiliar os sujeitos no desenvolvimento de habilidades para uma melhor qualidade de vida (Lohr et al., 2013).

Apesar da importância da prevenção primária e promoção de saúde na infância, e da existência de programas dessa natureza reconhecidos internacionalmente, esses ainda são pouco conhecidos, utilizados e estudados no Brasil (Abreu et al., 2015). As autoras ainda destacam a falta de pesquisas e incentivo a esses tipos de programas, bem como sua baixa inserção dentro das políticas públicas, contexto esse que poderia possibilitar resultados positivos e potencializados para grande parte da população.

Em consonância com as autoras, Figueiredo (no prelo) destaca que mesmo ao redor do mundo, tais práticas ainda são incipientes, porém, observam-se diversos programas de promoção de saúde baseado em evidências. Grande parte desses programas busca o desenvolvimento de "habilidades para vida" (life skills). As habilidades para vida, conforme Gorayeb (2002), são reconhecidas pela Organização Mundial de Saúde como competências adaptativas e adequadas para as crianças e adolescentes lidarem com os desafios e dificuldades do cotidiano. De acordo com esse mesmo autor, a OMS destaca algumas delas, como resolução de problemas, pensamento criativo, habilidades sociais, habilidade de tomada de decisão, autoconhecimento, hábitos de vida saudável (alimentação e atividade física) e manejo de emoções. Essas competências são de extrema importância ao longo do ciclo vital do sujeito, e sua construção busca promover o próprio desenvolvimento, com um aumento dos comportamentos saudáveis e uma diminuição dos comportamentos problemáticos (Botvin \& Griffin, 2015, p. 406).

Além disso, as habilidades para a vida podem se constituir como promotoras de resiliência. Segundo Papalia e Feldman (2013) e Yunes (2003), a resiliência pode ser caracterizada como a capacidade da criança de se recuperar e superar as situações estressantes, de medo e de adversidade de uma forma positiva. Porém, de acordo com Yunes (2015), essa adaptação e superação das adversidades não deve ser entendida somente como algo individual, deve ser entendida como um aspecto que é relacionado com forças e fatores de ordem social, histórica, política e cultural do sujeito e que atravessam os processos de desenvolvimento de sua vida. Para Papalia e Feldman (2013), as crianças resilientes resistem a situações adversas, enfrentam os desafios, os eventos novos ou estressantes, conseguem reunir forças para superar os obstáculos. Por isso são tão relevantes programas que possibilitem à criança o desenvolvimento das habilidades para a vida de uma forma lúdica e contextualizada, visto de seu potencial como fator de proteção no desenvolvimento infantil e de prevenção aos transtornos de ansiedade e depressão.

\section{Método FRIENDS}

Dentre os programas reconhecidos mundialmente para promoção de saúde e prevenção de ansiedade e depressão está o Método Friends (Barrett, Shortt, Fox, \& Wescombe, 2001; Barrett \& Turner, 2001; Stallard et al., 2005; Kösters et al., 2012). Este programa australiano foi construído por Paula Barrett e traduzido para o português-brasileiro em 2012. Ele tem como objetivo desenvolver habilidades para a vida: habilidades socioemocionais, resiliência, comportamentos positivos e hábitos de vida saudável. Refere-se a uma forma de intervenção em saúde mental baseada em evidências científicas, em técnicas cognitivo-comportamentais e também com aportes da psicologia positiva e da neurociência. Figueredo (no prelo) aponta que o Método busca desenvolver com as crianças e jovens estratégias para enfrentamento de estresse, com foco nas respostas cognitivas, fisiológicas e comportamentais. O Método possui ainda, baseado no lastro cognitivista, o trabalho com estratégias de reconhecimento de pensamentos, emoções e comportamento, bem como a reestruturação cognitiva, a partir do reconhecimento e questionamento de pensamentos falsos e incompatíveis e a possibilidade de gerar interpretações alternativas. Do lastro comportamental, busca o desenvolvimento de habilidade e competências sociais como o treino assertivo e de empatia, resolução de problemas, entre outros. 
O Método Friends apresenta-se como um programa estruturado e organizado para diferentes idades, possuindo quatro formas: Fun Friends (4-7 anos), Friends for Life (8-11 anos), My Friends (12-16 anos), Adult Resilience (17-em diante). Para cada faixa etária existe um material/manual organizado para o participante e também um para o facilitador responsável por mediar às intervenções (Barrett, 2012).

O nome FRIENDS vem de uma analogia com a própria palavra "amigos" (em inglês) que busca mostrar que fazer amigos, ter a si próprio como amigo e ser um bom amigo pode ajudar a criança a ser mais feliz. Essa analogia também se refere à sequência de encontros e suas respectivas habilidades que são treinadas com as crianças. A sequência de habilidades é estabelecida da seguinte forma: A - atenção aos sentimentos; $\mathrm{M}$ momentos de relaxar; I - ideias positivas; $\mathrm{G}$ - gerar soluções; $\mathrm{O}$ - orgulhe-se de seu trabalho; $\mathrm{S}$ - sorria e lembre-se de ser corajoso (Figueredo, no prelo; Iizuka \& Barrett, 2011).

O Friends é revisto periodicamente a cada três anos, sendo anexados a ele novos treinos e ajustados os que já existem. Ele está organizado em 14 encontros de 90 a 120 minutos: 10 encontros para grupos de crianças/ jovens; 2 encontros de reforço sobre as habilidades que foram aprendidas; 2 encontros para a comunidade (pais, cuidadores, professores, etc). Cada encontro possui uma sequência básica de competências e habilidades exploradas. Segundo Figueredo (no prelo), cada encontro possui um conteúdo próprio e está esquematizado da seguinte forma: $1^{\circ}$ encontro - apresentação do programa e as habilidades que serão trabalhadas nele aos participantes; $2^{\circ}$ encontro - desenvolvimento de habilidades de regulação emocional, identificação de sentimentos e percepção de sintomas fisiológicos; $3^{\circ}$ encontro - aprendizado sobre confiança e treino de habilidades sociais; $4 \mathrm{O}$ encontro - treino de relaxamento e Mindfulness para ampliar a atenção da criança para si e manejo das emoções; 5o encontro - compreensão dos pensamentos e sua ligação com seus sentimentos e comportamentos; 6 e encontro - foco nos sentimentos e habilidades de enfrentamento a diversos contextos e situações; $7 \underline{0}$ encontro - desenvolver habilidades de planejamento e resolução de problemas a partir do plano passo-a-passo; 8 o encontro - planos de resolução de problemas com uma rede de apoio e recursos; $9^{\circ}$ encontro - habilidades e estratégias de resolução de conflito e assertividade; $10^{\circ}$ encontro - apanhado geral de tudo que foi trabalhado durante o programa.

É importante apontar que esse programa está inserido dentro da abrangência de prevenção primária, buscando a promoção e construção de habilidades para a vida. Também pode ser classificada dentro do âmbito da prevenção universal que se trata, segundo Iizuka e Barrett (2011), de uma prevenção visando alcançar o público geral de crianças, independente de fatores de risco ou da presença de sintomas e promovendo um ambiente de aprendizagem e desenvolvimento para as crianças e para sua socialização.

Essa intervenção vem sendo pesquisada de modo científico em 13 países e utilizada em mais de 20 países, em alguns, inclusive, dentro das políticas públicas. É um programa estruturado de prevenção universal, que foi reconhecido pela OMS como eficaz no fortalecimento de habilidades socioemocionais, resiliência e na prevenção de transtornos relacionados à ansiedade e depressão (WHO, 2004). Além disso, ele vem sendo aplicado em diversos países tendo resultados positivos quanto sua eficácia e efetividade (Stallard et al., 2007; Kosters et al., 2012; Iizuka, Barrett, Gillies, Cook \& Miller, 2014). Segundo Vianna, Campos e Fernandez (2009) o programa Friends é considerado, ao redor do mundo, o programa de maior efetividade na prevenção de ansiedade. Diante do exposto, observase que o Método Friends figura como uma ótima ferramenta para a promoção de saúde e prevenção de ansiedade. Nesse contexto, o objetivo desse trabalho foi avaliar a eficácia do Método Friends em um grupo de crianças de uma cidade do Interior do Paraná. Pode-se considerar a relevância dessa avaliação do programa no Brasil, tanto pelo fato de haver escassez de pesquisas dentro da temática de prevenção e promoção de saúde na infância a partir de métodos estruturados, bem como pelo Método Friends ainda ter poucos estudos e pesquisas sobre sua eficácia no país.

\section{Método}

\section{Participantes}

Os participantes desta pesquisa foram 10 crianças, de ambos os sexos, com idades entre 6 e 7 anos. Este projeto foi aprovado pelo Comitê de Ética em Pesquisa da Universidade (Aprovação Comitê de Ética $\mathrm{n}^{\mathrm{O}}$ 55858916.1.0000.0106), tendo seguido todas as normas éticas referentes à pesquisa com seres humanos. Após a aprovação do Projeto pelo Comitê de ética, entrou-se em contato com a Clínica Escola da universidade para verificar se haviam crianças com idade entre 6 e 7 anos na fila de espera para atendimento psicoterápico. Havia apenas uma criança que preenchesse o critério de idade. Os pais da criança foram convidados a participar de uma primeira entrevista, onde foi apresentado o Método e a partir de seu aceite, esta foi incluída na pesquisa. Na sequência, foi entrado em contato com as escolas do município para a divulgação do Método Friends e convite para 
a participação das crianças. Os primeiros nove pais que demonstraram interesse na participação foram incluídos no estudo.

\section{Instrumento}

Para esse trabalho utilizou-se o CBCL 6-18 (Child Behavior Check List, Achenbach \& Rescorla, 2001), uma escala de verificação comportamental respondida por pais /cuidadores de crianças e adolescentes. O CBCL foi adaptado e validado para a população brasileira por Bordin, Mari e Caieiro (1995), tendo sido recentemente revalidado (Brasil \& Bordin, 2010), apresentando boas qualidades psicométricas de validade e confiabilidade (Rocha et al, 2013; Silvares, Rocha \& Emerich, 2016). O CBCL 6-18 anos é composto por duas partes, sendo a primeira a avaliação de competência social (sociabilidade, atividade e escolaridade) e a segunda a avaliação dos problemas emocionais/ comportamentais. Essa é formada a partir da a) escala de internalização, derivada do agrupamento das subescalas ansiedade/depressão, retraimento/depressão e queixas somáticas e b) da escala de externalização, corresponde ao agrupamento de problemas sociais, comportamento de quebrar regras e comportamento agressivo. As sub-escalas problemas de pensamento e problemas de atenção, junto a escala de internalização e escala de externalização compõem a escala do total de problemas emocionais/ comportamentais. Na seção de avaliação de competências sociais, a partir de 20 itens, os pais/cuidadores devem comparar o comportamento da criança com o comportamento de crianças da mesma idade quanto a tempo e desempenho despendido na ação descrita, apontando como abaixo da média, na média ou acima da média aquela ação. A seção de problemas de comportamento é composto por 118 itens, aos quais os pais devem considerar os últimos seis meses e responder a partir de uma escala likert de 3 pontos o quão verdadeiro é aquela descrição sobre a criança. Os resultados ponderados no CBCL permitem classificar as crianças em: não-clínica, limítrofe e clínica (Bordin, Mari \& Caiero, 1995). Para as escalas de competências sociais, é considerado clínicos escore abaixo de 31 e não clínico acima de 31 . Para as escalas de problemas emocionais/comportamentais, são considerados clínico escores acima de 63 e não clínico inferior a 60. Esse instrumento é avaliado por meio de análise computadorizada realizada através do software ADM 7.0. (Moura, Casanova, Meurer \& Campana, 2008).

\section{Procedimento}

Após a aprovação do Projeto pelo Comitê de Ética, e com a seleção dos participantes, os pais das crianças foram convidados para uma entrevista inicial, onde foram apresentados o Método Friends e a pesquisa. Após o aceite e assinatura do TCLE, foi aplicado o CBCL em pré-intervenção aos pais individualmente. Após essa etapa, as crianças participaram dos 10 encontros do Método, os quais seguiram a estrutura proposta por Barrett (2012). Ocorreram ainda dois encontros estruturados com os pais realizados em grupo, concomitante ao primeiro e $5^{\mathrm{O}}$ encontro das crianças, conforme prevê o Método. Os participantes foram novamente avaliados pelos pais, em pós-intervenção, com o mesmo instrumento. Posterior a essa avaliação, houve a aplicação de mais 2 encontros do Método, reforço 1 e reforço 2 , também seguindo a proposta de Barrett (2012). Por fim, foi feita avaliação follow up 1, que ocorreu 60 dias após o pós-teste, logo após o reforço 2.

\section{Análise de dados}

A metodologia empregada nesse trabalho é a quantitativa. A análise dos dados foi realizada a partir do software SPSS (Statistical Package for the Social Sciences) 17.0. Foi utilizada estatística descritiva e inferencial. Para a definição das estatísticas inferenciais utilizadas na análise de dados foi realizada uma avaliação da normalidade dos dados coletados no estudo. Para tanto foi utilizada a variável total de problemas emocionais/comportamentais do CBCL, considerando sua representatividade ao estudo, uma vez que essa é derivada de todas as escalas de problemas de comportamento. O Bloxplot desta variável indicou a ausência de outliers e o teste de normalidade de Shapiro-Wilk resultou em uma estatística de 0,95 $(\mathrm{gl}=10 ; \mathrm{p}=0,71)$, o que poderia sugerir uma distribuição normal da variável. No entanto, os valores de média, mediana e moda da variável apresentaram uma grande disparidade (média $=59,6$; mediana $=61 ;$ moda $=51$ ), o que condiz com uma distribuição assimétrica dos dados. Considerando isto e o tamanho reduzido da amostra, optou-se pela utilização de estatística não-paramétrica durante para a realização das análises de dados.

Para a comparação dos dados em pré e pós-teste, pós-teste e follow up, e pré-teste e follow up, foi utilizado o teste estatístico não-paramétrico de Wilcoxon.

\section{Resultados}

A avaliação da eficácia do Método Friends foi realizada a partir dos resultados do CBCL. A aplicação do instrumento foi realizada em três momentos: pré-teste, pós-teste e follow up. Nas avaliações do pré e do pós-teste participaram dez crianças, já na avaliação de follow up, apenas oito crianças. As Médias e o Desvio Padrão das escalas e sub-escalas do CBCL em cada uma das etapas de avaliação podem ser visualizadas na Tabela 1. 
TABELA 1

Descrição de Média e Desvio Padrão das escalas e sub-escalas do CBCL (Child Behavior Check List) nas três etapas de avaliação.

\begin{tabular}{|c|c|c|c|c|c|c|}
\hline \multirow{2}{*}{ Escalas e Sub-escalas } & \multicolumn{2}{|c|}{ Pré-teste } & \multicolumn{2}{|c|}{ Pós-Teste } & \multicolumn{2}{|c|}{ Follow Up } \\
\hline & Média & Desvio $P$ & Média & Desvio $P$ & Média & Desvio $P$ \\
\hline Atividade & 41,40 & 8,044 & 45,70 & 5,945 & 46,13 & 7,180 \\
\hline Sociabilidade & 49,10 & 6,118 & 52,10 & 8,319 & 55,38 & 6,501 \\
\hline Escolaridade & 48,50 & 8,923 & 47,10 & 7,978 & 49,50 & 7,290 \\
\hline Competências Totais & 45,30 & 7,040 & 49,20 & 8,664 & 52,63 & 7,444 \\
\hline Ansiedade/ Depressão & 64,40 & 3,836 & 58,20 & 5,051 & 52,25 & 3,012 \\
\hline Retraimento/Depressão & 60,10 & 8,950 & 57,60 & 6,022 & 53,25 & 3,991 \\
\hline Queixas Somáticas & 57,70 & 6,255 & 55,20 & 5,203 & 54,88 & 5,222 \\
\hline Problemas Sociais & 56,80 & 5,846 & 54,00 & 5,055 & 54,13 & 4,643 \\
\hline Problemas de Pensamento & 55,00 & 6,394 & 54,20 & 5,827 & 51,63 & 2,925 \\
\hline Problemas de Atenção & 59,50 & 13,746 & 54,20 & 4,662 & 54,88 & 7,643 \\
\hline Comportamento de Quebrar Regras & 58,10 & 7,125 & 52,70 & 3,164 & 51,63 & 1,847 \\
\hline Comportamento Agressivo & 60,90 & 8,386 & 55,10 & 5,131 & 51,13 & 1,458 \\
\hline Escala de Internalização & 62,90 & 6,027 & 57,60 & 5,661 & 49,13 & 8,823 \\
\hline Escala de Externalização & 59,10 & 10,333 & 51,20 & 8,879 & 44,63 & 8,158 \\
\hline Total de Problemas Emocionais/Comportamentais & 59,60 & 10,124 & 52,80 & 5,633 & 46,75 & 10,039 \\
\hline
\end{tabular}

A partir da Tabela 1 pode-se perceber um aumento na maioria das médias das sub-escalas que compõem a escala de competências sociais ao longo do processo; nas sub-escalas atividade, sociabilidade, competências totais, todas as médias tiveram um aumento em aceleração positiva da avaliação pré-teste para o follow up. Somente na sub-escala escolaridade observa-se uma pequena diminuição do pré para o pós-teste, voltando a aumentar as médias no follow up.

Em relação às médias das escalas de internalização, externalização e dos problemas de pensamento, observou-se uma aceleração negativa nos três momentos de avaliação, ou seja, escores mais altos no pré-teste decaindo de forma progressiva até o pósteste. Outras escalas também apresentaram resultado semelhante. Os problemas de atenção tiveram uma diminuição no pré-teste para o pós-teste, com um pequeno aumento no follow up.

Buscando-se verificar se as mudanças observadas na análise descritiva foram significativas, procedeu-se a análise estatística inferencial a partir do teste nãoparamétrico de Wilcoxon. Esse teste é utilizado para comparação de amostras pareadas, comparando-se dois momentos de avaliação de um mesmo sujeito. A partir dessa comparação tem-se o escore Z. Tomou-se como significativas diferenças com $p \leq 0,05$. Destacase que na comparação pré e pós-teste, contou-se com um $n=10$. Já na comparação pré-teste e follow-up e pós-teste e follow up teve-se um $\mathrm{n}=8$. Os resultados podem ser observados na Tabela 2.
TABELA 2

Análise inferencial de comparação não paramétrica de Wilcoxon da amostra a partir do CBCL (Child Behavior Check List).

\begin{tabular}{lccc}
\hline Escalas e Sub-escalas & Pré-Pós & Pós-Follow & Pré-Follow \\
\hline Atividades & $\mathrm{Z}=-1,562$ & $\mathrm{Z}=-1,807$ & $\mathrm{Z}=-1,352$ \\
& $\mathrm{p}=0,11$ & $\mathrm{p}=0,07$ & $\mathrm{p}=0,17$ \\
Social & $\mathrm{Z}=-1,365$ & $\mathrm{Z}=-0,848$ & $\mathrm{Z}=-2,033$ \\
& $\mathrm{p}=0,17$ & $\mathrm{p}=0,39$ & $\mathrm{p}=0,04^{*}$ \\
Escolar & $\mathrm{Z}=-1,409$ & $\mathrm{Z}=-0,677$ & $\mathrm{Z}=-1,000$ \\
& $\mathrm{p}=0,15$ & $\mathrm{p}=0,49$ & $\mathrm{p}=0,31$ \\
Competências Totais & $\mathrm{Z}=-1,429$ & $\mathrm{Z}=-1,198$ & $\mathrm{Z}=-1,960$ \\
& $\mathrm{p}=0,15$ & $\mathrm{p}=0,23$ & $\mathrm{p}=0,05^{*}$ \\
Ansiedade/ Depressão & $\mathrm{Z}=-2,075$ & $\mathrm{Z}=-2,213$ & $\mathrm{Z}=-2,524$ \\
& $\mathrm{p}=0,03^{*}$ & $\mathrm{p}=0,02 *$ & $\mathrm{p}=0,01^{*}$ \\
Retraimento/Depressão & $\mathrm{Z}=-1,039$ & $\mathrm{Z}=-1,604$ & $\mathrm{Z}=-2,456$ \\
& $\mathrm{p}=0,29$ & $\mathrm{p}=0,10$ & $\mathrm{p}=0,01^{*}$ \\
Queixas Somáticas & $\mathrm{Z}=-1,022$ & $\mathrm{Z}=-0,742$ & $\mathrm{Z}=-1,289$ \\
& $\mathrm{p}=0,30$ & $\mathrm{p}=0,45$ & $\mathrm{p}=0,19$ \\
Problemas Sociais & $\mathrm{Z}=-2,201$ & $\mathrm{Z}=-0,843$ & $\mathrm{Z}=-0,422$ \\
& $\mathrm{p}=0,02^{*}$ & $\mathrm{p}=0,39$ & $\mathrm{p}=0,67$ \\
Problemas de Pensamento & $\mathrm{Z}=-0,508$ & $\mathrm{Z}=-1,826$ & $\mathrm{Z}=-1,414$ \\
& $\mathrm{p}=0,61$ & $\mathrm{p}=0,06$ & $\mathrm{p}=0,15$ \\
Problemas de Atenção & $\mathrm{Z}=-1,825$ & $\mathrm{Z}=-0,420$ & $\mathrm{Z}=-1,265$ \\
& $\mathrm{p}=0,06$ & $\mathrm{p}=0,67$ & $\mathrm{p}=0,20$ \\
Comportamento de & $\mathrm{Z}=-2,371$ & $\mathrm{Z}=-0,730$ & $\mathrm{Z}=-1,782$ \\
Quebrar Regras & $\mathrm{p}=0,01^{*}$ & $\mathrm{p}=0,46$ & $\mathrm{p}=0,07$ \\
Comportamento Agressivo & $\mathrm{Z}=-2,524$ & $\mathrm{Z}=-2,041$ & $\mathrm{Z}=-2,375$ \\
& $\mathrm{p}=0,01^{*}$ & $\mathrm{p}=0,04^{*}$ & $\mathrm{p}=0,01^{*}$ \\
Escala de Internalização & $\mathrm{Z}=-1,943$ & $\mathrm{Z}=-2,100$ & $\mathrm{Z}=-2,524$ \\
& $\mathrm{p}=0,05^{*}$ & $\mathrm{p}=0,03 *$ & $\mathrm{p}=0,01^{*}$ \\
Escala de Externalização & $\mathrm{Z}=-2,809$ & $\mathrm{Z}=-1,706$ & $\mathrm{Z}=-2,380$ \\
& $\mathrm{p}=0,00^{*}$ & $\mathrm{p}=0,08$ & $\mathrm{p}=0,01^{*}$ \\
Total de Problemas & $\mathrm{Z}=-2,552$ & $\mathrm{Z}=-1,947$ & $\mathrm{Z}=-2,527$ \\
Emocionais/ Comportamentais & $\mathrm{p}=0,01^{*}$ & $\mathrm{p}=0,05^{*}$ & $\mathrm{p}=0,01^{*}$ \\
\hline & & & \\
a & & &
\end{tabular}

$* \mathrm{p} \leq 0,05$. 
Pode-se observar a partir da Tabela 2 que foram pareados os três momentos da avaliação, para fins de comparação. Foi observado diferença estatisticamente significativa do pré-teste para of follow up em uma escala e duas sub-escalas: desempenho social, retraimento/ depressão e na escala de competências totais.

Diferenças estatisticamente significativas ocorreram do pré-teste para o pós-teste nas sub-escalas problemas sociais e comportamento de quebrar regras. $\mathrm{Na}$ escala de externalização, foram significativas as diferenças de escores do pré-teste para o pós-teste e do pré-teste para follow up.

Por fim, foram observadas diferenças estatisticamente significativas entre os três momentos de avaliação nas sub-escalas ansiedade/depressão e comportamento agressivo. Também na escala de internalização e escala do total de problemas emocionais/ comportamentais.

\section{Discussão}

O Método Friends é um programa de promoção de saúde e prevenção de transtornos de ansiedade e transtornos depressivos na infância, que, segundo Iizuka e Barrett (2011), ocorre por meio da promoção de habilidades de enfrentamento, de comportamentos positivos e também pelo aumento de resiliência emocional. Diante disso, o objetivo deste trabalho foi a avaliação da eficácia do Método Friends, em um grupo de 10 crianças com idades entre de 6 e 7 anos de uma cidade do interior do Paraná. O instrumento utilizado para avaliação da eficácia foi o CBCL.

O CBCL é composto por duas partes: uma relacionada à avaliação de competências sociais e outra de problemas de comportamento. Na primeira seção são avaliadas competências sociais e habilidades que são o objetivo do Método. Na segunda parte, são verificadas dificuldades comportamentais da criança que podem melhorar com o desenvolvimento de habilidades propostas pelo Método Friends. Na escala de competências sociais são avaliadas, por exemplo, questões relacionadas às habilidades sociais da criança, habilidades de fazer amigos e de se relacionar com outras crianças. O Método Friends tem como um de seus objetivos o desenvolvimento de habilidades sociais: fazer amizades, reconhecer os sentimentos em si e nos outros, ser empático e se autorregular (Iizuka \& Barrett, 2011).

Pode-se verificar, a partir dos resultados na escala de competência social, o quanto, a partir da visão dos pais, as crianças tiveram um aumento dessas habilidades após a intervenção, o que pode indicar que as crianças aprimoraram tais competências que podem ser facilitadoras de suas relações sociais (Figueredo, no prelo). Cabe ressaltar que essas habilidades, tidas como habilidades socioemocionais, são consideradas na literatura (Figueredo \& Torres, 2016; Abreu et al., 2015), como promotoras de saúde mental, pois são competências relacionadas ao reconhecimento e manejo de emoção, empatia, tomada de decisão, manejo de situações problema, que podem auxiliar a criança a enfrentar as dificuldades do cotidiano.

Quanto a seção Problemas de Comportamento do CBCL, tem-se a escala de internalização, que se refere aos comportamentos das crianças ligados a sintomas de ansiedade, depressão, retraimento, tristeza e queixas somáticas e de irritabilidade. Nessa escala observou-se decréscimo dos escores nos três momentos de avaliação, com diferenças estatisticamente significativas. Segundo alguns autores a ansiedade e depressão possuem sintomatologia (Fernandes et al., 2014; Fraga, 2015; Bahls, 2002; Vianna et al., 2009) e fatores de risco (Iizuka \& Barrett, 2011; Maia \& Williams, 2005; Figueiredo, no prelo) similares. Nesse contexto, intervenções que ajam sobre esses transtornos devem produzir uma redução desses fatores e da frequência dos comportamentos que são indicativos dos mesmos. O Método Friends desenvolve habilidades, tais como ficar atento aos seus sentimentos, conhecê-los, falar sobre eles e reconhecê-los nos outros; habilidades de empatia, estratégias de relaxamento e de respiração, habilidades de reconhecimento de pensamentos disfuncionais (pensamentos vermelhos em verdes), resolução de problemas, entre outros (Iizuka \& Barrett, 2011; Figueredo, Nico \& Leonardi, 2015, Stallard et al., 2010), que são descritos na literatura como fatores de proteção ao desenvolvimento dos sintomas de ansiedade e depressão (Stallard et al., 2010).

Os resultados do presente estudo apontaram para uma diminuição significativa em todas as escalas e subescalas do CBCL relacionadas à escala de internalização, podendo supor a eficácia do Método Friends quando à construção de habilidades e competências que contribuem para o manejo e prevenção de ansiedade e depressão na presente amostra. Estudos sobre a avaliação do Método Friends na Inglaterra (Stallard et al., 2005; Stallard, Taylor et al., 2014) demonstram que o programa foi efetivo e diminuiu sintomas de ansiedade em crianças, sendo que os resultados se mantiveram em follow up de 24 meses. Segundo Fernandes, Alckmin-Carvalho, Izbicki e Melo (2014), a partir de revisão sistemática sobre programas de prevenção à ansiedade na infância e na adolescência, o Método Friends é o programa de prevenção universal de ansiedade mais aplicado e avaliado no mundo, sendo o método mais robusto e com resultados mais 
significativos no que tange à prevenção à ansiedade. Os resultados do presente estudo vão ao encontro de outros estudos (Iizuka \& Barrett, 2011; Figueredo, Nico, \& Leonardi, 2015) que também demonstraram que crianças que apresentavam elevados níveis de ansiedade antes do programa diminuíram os sintomas após o término da intervenção.

A escala de externalização do CBCL corresponde ao agrupamento das sub-escalas problemas sociais, comportamento de quebrar regras e comportamento agressivo; essas podem ser traduzidas nas dificuldades de fazer amigos, dificuldade de autocontrole, dificuldade nas relações sociais e seguimento de regras (Borsa \& Nunes, 2008). Pode-se observar que no Método Friends são realizadas atividades que visam aumentar as habilidades sociais das crianças, bem como estratégias de autorregulação emocional (Figueredo, no prelo). Também compõe o Método o foco no desenvolvimento de habilidades de resolução de problemas, habilidades de se auto-recompensar pelo esforço e fortalecimento de redes de apoio (Iizuka \& Barrett, 2011). A partir dos resultados apresentados pode-se observar uma diminuição dos escores de PE do início do programa para o fim do mesmo, indicando um possível incremento de habilidades e competências envolvidas na regulação dos comportamentos externalizantes e aumento de comportamentos pró-sociais. Esses dados vão ao encontro de outros estudos com o Método Friends que demostram um aumento de comportamentos prósociais e diminuição de problemas de relacionamento interpessoal nas crianças após a participação no programa (Stopa, Barrett, \& Golingi, 2010; Anticich, Barrett, Silverm, Lacherez, \& Gillies, 2013; Ahlen, Breithaltz, Barrett, \& Gallegos, 2012). Stopa et al., (2010) apontaram que essa diminuição de problemas pode estar relacionada a um aumento da autoestima das crianças, sugerindo que a partir da participação no Método as crianças desenvolvem repertórios que contribuiriam para suas relações interpessoais, como habilidades de resolução de problemas e de regulação emocional, tendo como consequência, maior aceitação e relacionamento positivo com pares.

Cabe ressaltar que o Método conta com encontros com os pais e com a comunidade na qual a criança está inserida. Nesses encontros busca-se ensinar habilidades aos cuidadores que possam contribuir para a generalização dos comportamentos que a criança aprende a partir das intervenções do Friends, o que também é uma variável que pode influenciar os resultados e contribuir para sua manutenção no follow up.

Algumas sub-escalas (problemas de pensamento e problemas de atenção) não apresentaram diferenças estatisticamente significativas entre os três momentos de avaliação, apesar de terem apresentado decréscimo em seus escores do início para o final da intervenção. A média da sub-escala problemas de pensamento já se apresentava baixa ao início da intervenção, e assim manteve-se nos três momentos. Já a subescala problemas de atenção apresentava um elevado desvio-padrão na avaliação pré-teste, o que indica que provavelmente poucos participantes apresentaram escore elevado nessa sub-escala ao início do Programa. Ao longo da intervenção esse desvio padrão diminui. É possível que, em uma análise de caso único, seja possível verificar a eficácia do Método sobre essa queixa.

Por fim, a escala de total de problemas emocionais/comportamentais apresentou diferenças estatisticamente significativas entre os três momentos de avaliação. Essa escala é formada a partir das escalas de internalização e externalização, adicionadas das subescalas de problemas com o pensamento e problemas de atenção. Como apontado anteriormente, as escalas de internalização e de externalização tiveram grandes diferenças nas médias dos escores nos três momentos de avaliação, o que pode explicar as diferenças em TP. Este pode ser considerado um indicativo de quanto às habilidades desenvolvidas no Método Friends têm um efeito global de incremento de repertórios comportamentais em seus participantes, que se reflete na diminuição de sintomatologias. Todos esses dados apoiam a eficácia do Método na população avaliada.

\section{Considerações finais}

Esta pesquisa teve por objetivo verificar a eficácia do Método Friends, em um grupo de crianças com idades entre 6 e 7 anos, residentes em uma cidade no Interior do Paraná. Os resultados demonstraram aumento de competências socioemocionais dos participantes, bem como redução de comportamentos relacionados a problemas sociais e sintomas de ansiedade e depressão.

Apesar dos resultados promissores, esta pesquisa apresentou algumas restrições que interferem na possibilidade de generalização dos dados. Destaca-se o baixo número de participantes do grupo, o método quase-experimental utilizado, bem como os participantes serem residentes de uma cidade de pequeno porte do interior do Paraná. Assim, partindo disso, sugere-se a necessidade de mais pesquisas, que tenham como possibilidade ampliar o número de participantes, como desenvolver o Método em outros contextos, como em escolas e Centros de Referência da Assistência Social (CRAS), entre outros. 
O uso de Metodologias Experimentais e de Estudos Clínicos Randomizados também seriam interessantes para verificação de eficácia do Método no contexto brasileiro.

Conclui-se que, diante dos resultados obtidos no presente estudo, o Método Friends apresenta bons indícios de eficácia para a promoção de habilidades socioemocionais e prevenção de ansiedade e depressão. Esses dados vão ao encontro de estudos internacionais (Iizuka \& Barrett, 2011; Stallard et al., 2005; 2008; Stallard, 2010) que apontam para a eficácia do Método Friends, destacando-se seu potencial de prevenção e promoção de saúde e a possibilidade de ser utilizado em larga escala.

\section{Referências}

Abreu, S., Barletta, J. B., \& Murta, S. G. (2015). Prevenção e promoção em saúde mental: pressupostos teóricos e marcos conceituais. In Murta, S. G; França, C. L; Santos, K. B; Polejack, L. Prevenção e promoção em saúde mental: fundamentos, planejamento e Estratégias de intervenção (pp. 54-74). Novo Hamburgo: Sinopsys.

Achenbach, T. M. \& Rescorla, L. A. (2001). Manual for the ASEBA school-age forms \& profiles. Burlington, VT: University of Vermont. Department of Psychiatry.

Ahlen, J., Breithaltz, E., Barrett, P. M., \& Galegos, J. (2012) School -based prevention of anxiety and depression a piloto study in sweden. Advances in school mental health promotion, 5(4), 246 -257. https://doi.org/10.1080/17547 30X.2012.730352

American Psychiatric Association. (2014). DSM-5: manual diagnóstico e estatístico de transtornos mentais (5a ed.). Porto Alegre: Artmed.

Anticich, S. A., Barrett, P. M., Silverm, W., Lacherez, P., \& Gillies, R. (2013). The prevention of childhood anxiety and promotion of resilience among preschool-aged children: a universal school based trial. Advances in school mental health promotion, 6(2), 93-121. https://doi.org/10.1080/1754730X.2013.784616

Bahls, S.C. (2002). Aspectos clínicos da depressão em crianças e adolescentes. Jornal de Pediatria, 78(5), 359-366. https://doi.org/10.1590/S0021-75572002000500004

Bahls, S. C. (2002). Epidemiology of depressive symptoms in adolescents of a public school in Curitiba, Brazil. Revista Brasileira de Psiquiatria, 24 (2), 63-67. https://doi.org/10.1590/s1516-44462002000200005

Barrett, P. M. (2012). Amigos Divertidos: Guia do Facilitador para construção de resiliência em crianças de 4 a 7 anos através do brincar (3aㅡ ed.). Austrália: Barrett Research Resources PTy Ltd.

Barrett, P. M., Shortt, A. L., Fox, T. L., \& Wescombe, K. (2001). Examining the social validity of the FRIENDS treatment program for anxious children. Behavior Change, 18(2), 63-77. https://doi.org/10.1375/bech.18.2.63

Barrett, P. M. \& Turner, C. (2001). Prevention of anxiety symptoms in primary school children: Preliminary results from a universal school - based trial. British Journal of Clinical Psychology, 40, 399-410. https://doi. org/10.1348/014466501163887

Bordin, I. A. S., Mari, J. J., \& Caieiro, M. F. (1995). Validação da versão brasileira do Child Behavior Checklist (CBCL) Inventário de comportamentos da Infância e adolescência: dados preliminaries. Revista ABP APPAL, 17(921), 55-66.

Borsa, J. C. \& Nunes, M. L. T. (2008). Concordância parental sobre problemas de comportamento infantil através do CBCL. Paidéia, 18(40), 317-330. http://dx.doi.org/10.1590/S0103-863X2008000200009

Botvin, G. J. \& Griffin, K. W. (2015). Treinamento de habilidades para a vida. In Murta, S. G., França, C. L., Santos, K. B., \& Polejack, L. Prevenção e promoção em saúde mental: fundamentos, planejamento e Estratégias de intervenção (pp. 405-418). Novo Hamburgo: Sinopsys.

Brasil, H. H. \& Bordin, I. A .S. (2010). Convergent validity of K-SADS-PL by comparison with CBCL in a Portuguese speaking outpatient population. BMC Psythiatry, 10, 83. https://doi.org/10.1186/1471-244X-10-83

Cia, F. \& Barham, E. J. (2009). Repertório de habilidades sociais, problemas de comportamento, autoconceito e desempenho acadêmico de crianças no início da escolarização. Estudos de. Psicologia, 26(1), 45-55. https://doi. org/10.1590/S0103-166X2009000100005

Fernandes, L. F. B., Carvalho, F. A., Izbicki, S., \& Melo, M. H. S. (2014) Prevenção Universal de Ansiedade na infância e adolescência: Uma revisão sistemática. Revista Psicologia: Teoria e Prática, 16(3), 83-99. São Paulo, SP. https:// doi.org/10.15348/1980-6906/psicologia.v16n3p83-99

Figueredo, L. Z. (No prelo) Uma luz no fim do túnel? Programas de prevenção e promoção de saúde mental e a implementação do Método FRIENDS no Brasil.

Figueredo, L. Z. (2015). Método Friends como estratégia de políticas públicas para prevenção em saúde mental e aproximações entre as abordagens cognitiva e analítico-comportamental. In X CBTC: Congresso Brasileiro de Terapia Cognitiva. Porto de Galinhas. Retirado de: <http://congresso.fbtc.org.br/admin/trabalhosCBTC/IAS XCBTC003.pdf>. Acesso em: jun. 2016. 
Figueredo, L. Z. \& Torres, L. (2016). Desenvolvimento de habilidades Socioemocionais na escola e a experiência bemsucedida do método Friends: quando as neurociências e a educação se encontram pelo caminho da psicologia positiva. In Barr, M. Neurociências e Educação na Primeira Infância: progressos e obstáculos (pp. 92-108) Brasília: Senado Federal. Comissão de Valorização da Primeira Infância e Cultura da Paz.

Figueredo, L. Z., Nico, Y., \& Leonardi, J. L. (2015). A experiência do Método Friends: uma possibilidade de prevenção e promoção de saúde mental em larga escala no Brasil. Boletim Paradigma, 10, 20-24.

Fraga, B. P. (2015). Depressão Infantil: Uma revisão da literatura. Universidade Federal do Rio Grande do Sul. Instituto de Psicologia. Curso de Especialização em Infância e Família: Avaliação, Prevenção e Intervenção. Porto Alegre.

Gorayeb, R. (2002). O ensino de habilidades de vida em escolas no Brasil. Psicologia, Saúde \& Doença, 3, $213-217$.

Iizuka, C. \& Barrett, P. (2011). Programa Friends para tratamento e prevenção de transtornos de ansiedade em crianças e adolescentes. In Petersen, C. S. et al. Terapias cognitivo comportamentais para crianças e adolescentes: ciência e arte (pp. 264-281). Porto Alegre: Artmed.

Iizuka, C. A., Barrett, P. M., Gillies, R, Cook, C. R., \& Miller, D. (2014). The FRIENDS Emocional Health Program for Minority Groups at Riso. J School Health, 84(2), 124-32. https://doi.org/10.1111/josh.12127

Kessler, R. C., Amminger, G. P., Aguilar-Gaxiola, S., Alonso, J., Lee, S., \& Ustün, T. B. (2007). Age of onset of mental disorders: a review of recent literature. Current opinion in psychiatry, 20(4), 359-64. https://doi.org/10.1097/ YCO.0b013e32816ebc8c

Kosters, M. P., Chinapaw, M. J., Zwaanswijk, M., Vander Wal, M. F., Mwjutens, E., \& Koot, H. (2012). M. Study design of 'FRIENDS for life': Process and effect evaluation of an indicated school-based prevention programme for childhood anxiety and depression. Bmc public health, 12(1), 86. https://doi.org/10.1186/1471-2458-12-86

Lohr, S., Pereira, A. C. S., Andrade, A. L. M., \& Kirchner, L. F. (2007) Avaliação de programas preventivos: relato de experiência. Psicologia em estudo, 12(3), 641-649. https://doi.org/10.1590/S1413-73722007000300022

Lohr, S. S., Melo, M. H. S., De Salvo, C. G., \& Silvares, E. F. M. (2013). Prevenção e promoção da Saúde: Um desafio na formação de psicólogos. Revista de psicologia da Criança e do Adolescente, 4(2), 205-222.

Maia, J. M. D. \& Williams, L. C. A. (2005). Fatores de risco e fatores de proteção ao desenvolvimento infantil: uma revisão da área. Temas em Psicologia, 13(2), 91-103.

Melnik, T. (2011). Psicologia Baseada em Evidências - Provas Científicas da Efetividade da Psicoterapia. São Paulo, Santos.

Menezes, J. C. L. (2013). Desenvolvimento positivo e saúde mental de crianças: Uma revisão sistemática de estudos brasileiros. Dissertação de Mestrado. Universidade de Brasília, Brasília.

Moura, C. B., Marinho-Casanova, M. L., Meurer, P. H., \& Campana, C. (2008). Caracterização da clientela pré-escolar de uma clínica-escola brasileira a partir do Child Behavior Checklist (CBCL). Contextos Clínicos, 1(1), 1-8.

Murta, S. G. (2005). Aplicações do Treinamento em Habilidades Sociais: Análise da Produção Nacional. Psicologia: Reflexão e Crítica, 18(2), 283-291. https://doi.org/10.1590/s0102-79722005000200017

Murta, S. G. (2007). Programas de prevenção a problemas emocionais e comportamentais em crianças e adolescentes: lições de três décadas de pesquisa. Psicologia: Reflexão e Crítica, 20(1), 01-08. https://doi.org/10.1590/s010279722007000100002

Murta, S. G., França, C. L., Santos, K. B., \& Polejack, L. (2015). Prevenção e promoção em saúde mental: fundamentos, planejamento e Estratégias de intervenção. Novo Hamburgo: Sinopsys.

Método Friends. Retirado de: <http://www.metodofriends.com/>. Acesso em: jul. 2015.

Organization, W. H. (2001). Mental Health: A Call to Action by World Health Ministers. WHO.

Organization, W. H. (2004). Prevention of mental disorders: effective interventions and policy options: summary report/a report of the World Health Organization Dept. of Mental Health and Substance Abuse; in collaboration with the Prevention Research Centre of the Universities of Nijmegen and Maastricht.

Organization, W. H. (2011). Mental health atlas. WHO.

Organization, W. H. (2012). Risks to Mental Health: an overview of vulnerabilities and risk factors. WHO.

Organization, W. H. (2014). World health statistics. WHO.

Papalia, D. E. \& Feldman, R. D. (2013). Desenvolvimento humano (12ª ed.). Porto Alegre: AMGH.

Rocha, M. M., Rescorla, L. A., Emerich, D. R., Silvares, E. F. M., Borsa, J. C., Araujo, L. S. et al. (2013). Behavioral/ Emotional problems in Brazilian children: findings from parents' report on the child behavior ckecklist. Epidemiol psychiattrc sciense, 22(4), 329-338. 10.1017/S2045796012000637

Salvo, C. G., Silvares, E. F. M., \& Toni, P. M. (2005). Práticas educativas como forma de predição de problemas de comportamento e competência social. Estudos de Psicologia, 22(2), 187-195. https://doi.org/10.1590/S0103166X2005000200008

Sampieri, R. H., Collado, C. F., \& Lucio, P. B. (2006). Metodología de la Investigación (4ạ ed.). México: McGraw Hill.

Silvares, E. F. M., Rocha, M. M.., \& Emerch, D. R. (2016). Inventario de comportamento da infância e da adolescência (CBCL), relatório para professores (TRF), inventario de autoavaliação para adolescentes (YSR). In Gorenstein, C., Wang, Y., Hungerbuhler, I. (Org.). Instrumentos de avaliação em Saúde Menta (pp. 331-336). Porto Alegre: Artmed. Stallard, P. (2010). Ansiedade: terapia cognitivo-comportamental para crianças e jovens. Porto Alegre: Artmed. 
Stallard, P. (2004). Bons Pensamentos-Bons Sentimentos: Manual da Terapia Cognitivo-Comportamental para Crianças e Adolescentes. Porto Alegre: Artmed.

Stallard, P. (2010). Mental health prevention in UK classrooms: the FRIENDS anxiety prevention programme. Emotional and Behavioural Difficulties, 15(1) 23-35. https://doi.org/10.1080/13632750903512381

Stallard, P., Simpson, N., Anderson, S., Carter, T., Osborn, C., \& Bush, S. (2005) An evaluation of the FRIENDS programme: a cognitive behaviour therapy intervention to promote emotional resilience. Archives of Disease in Childhood, 90(10), 1016-1019. https://doi.org/10.1136/adc.2004.068163

Stallard, P.; Simpson, N.; Anderson, S.; Anderson, S., Hibbert, S.; Osborn, C. (2007). The FRIENDS emotional health programme: Initial findings from a school-based project. Chile and Adolescent Mental Health, 12 (1), 32-37. https:// doi.org/10.1111/j.1475-3588.2006.00421.x

Stallard, P., Simpson, N., Anderson, S., \& Goddard, M. (2008). The FRIENDS emotional health prevention programme: 12 month follow up of a universal UK school based trial. European child \& adolescent psychiatry, 17(5), 283-289. https://doi.org/10.1007/s00787-007-0665-5

Stallard, P., Skryabina, E., Taylor, G., Phillips, R., Daniels, H., Anderson, R., \& Simpson, N. (2014). Classroom-based cognitive behaviour therapy (FRIENDS): a cluster randomised controlled trial to Prevent Anxiety in Children through Education in Schools (PACES), Lancet Psychiatry, 1, 185-92. https://doi.org/10.1016/S2215-0366(14)70244-5

Stallard, P., Taylor, G., Anderson, R., Daniels, H., Simpson, N., Phillips, R., \& Skryabina. (2014). The prevention of anxiety in children through school-based interventions: study protocol for a 24-month follow-up of the PACES project. Trials, 15, 77. https://doi.org/10.1186/1745-6215-15-77

Stopa, J. E., Barrett, P. M., \& Golingi, F. (2010). The prevention of childhood anxiety in socioeconomically disadvantaged communities: A universal school-based trial. Advances in School Mental Health Promotion, 3(4), 5-24. https://doi.or g/10.1080/1754730X.2010.9715688

Teste de Wilcoxon. Retirado de: <http://www.inf.ufsc.br/ vera.carmo/Testes_de_Hipoteses/Teste nao parametrico Wilcoxon.pdf>. Acesso em: nov. 2016.

Vianna, R. R. A. B., Campos, A. A., \& Landeira Fernandez, J. (2009). Transtornos de ansiedade na infância e adolescência: uma revisão. Revista Brasileira de Terapia Cognitiva, 5(1), 46-61.

Yunes, M. A. M. (2003). Psicologia Positiva e Resiliência: o foco no indivíduo e na família. Psicologia em Estudo (Maringá), 8, 75-84. http://dx.doi.org/10.1590/S1413-73722003000300010

Yunes, M. A. M. (2015). Dimensões conceituais da resiliência e suas interfaces com risco e proteção. In Murta, S. G., França, C. L., Santos, K. B., \& Polejack, L. Prevenção e promoção em saúde mental: fundamentos, planejamento e Estratégias de intervenção (pp. 93-112). Novo Hamburgo: Sinopsy.

\section{Agradecimentos:}

As autoras agradecem ao Instituto Brasileiro de Inteligência Emocional e Social -IBIES, representante internacional do Método FRIENDS no Brasil, pela parceria que possibilitou a realização dessa pesquisa.

Dados dos autores:

Giulia Tatiana Tkaczyk Pavoski - Acadêmica, Universidade Estadual do Centro-Oeste.

Caroline Guisantes de Salvo Toni - Doutora, Universidade Estadual do Centro-Oeste.

Ana Priscila Batista - Doutora, Universidade Estadual do Centro-Oeste.

Cynthia Lais Ignachewski - Acadêmica, Universidade Estadual do Centro-Oeste.

Endereço para correspondência:

Giulia Tatiana Tkaczyk Pavoski

Rua Dona Noca, 550

84500-000, Irati, PR, Brasil

<gt.pavoski@yahoo.com.br>

Recebido em: 31.01.2017

Aceito em: 21.12.2017 